\title{
Temperature Dependent Current-Voltage and Capacitance-Voltage Characteristics of an Au/n-Type Si Schottky Barrier Diode Modified Using a PEDOT:PSS Interlayer
}

\author{
Zagarzusem Khurelbaatar ${ }^{1}$, Kyu-Hwan Shim ${ }^{1}$, Jaehee Cho ${ }^{1}$, Hyobong Hong ${ }^{2}$, \\ V. Rajagopal Reddy ${ }^{3, *}$ and Chel-Jong Choi ${ }^{1, *}$ \\ ${ }^{1}$ School of Semiconductor and Chemical Engineering, Semiconductor Physics Research Center (SPRC), \\ Chonbuk National University, Jeonju 561-756, Republic of Korea \\ ${ }^{2}$ IT Convergence Technology Research Laboratory, Electronics \& Telecommunication Research Institute, \\ Daejeon 306-700, Republic of Korea \\ ${ }^{3}$ Department of Physics, Sri Venkateswara University, Tirupati-517 502, India
}

\begin{abstract}
The temperature dependence of the current-voltage (I- $V$ ) and capacitance-voltage $(C-V)$ characteristics of an Au/n-type Si Schottky barrier diode (SBD) with a PEDOT:PSS interlayer was investigated. The SBD parameters, such as Schottky barrier height $\left(\Phi_{\mathrm{B}}\right)$, ideality factor $(n)$, saturation current $\left(I_{0}\right)$, doping concentration $\left(N_{\mathrm{D}}\right)$, and series resistance $\left(R_{\mathrm{S}}\right)$, were obtained as a function of temperature. The Richardson constant $\left(A^{* *}\right)$ obtained from the $\operatorname{In}\left(I_{\mathrm{o}} / T^{2}\right)$ versus $1000 / T$ plot was much less than the theoretical value for n-Si. The mean Schottky barrier height $\left(\bar{\Phi}_{\text {bo }}\right)$ and standard deviation $\left(\sigma_{0}\right)$ calculated using the apparent Schottky barrier height $\left(\Phi_{\mathrm{ap}}\right)$ versus $1 / 2 \mathrm{kT}$ plot were $1.26 \mathrm{eV}$ and $0.15 \mathrm{eV}$, respectively. From a fit of the modified Richardson plot of $\ln \left(I_{0} / T^{2}\right)-(q \sigma)^{2} / 2(k T)^{2}$ versus $1000 / T$, the $A^{* *}$ was extracted as $134 \mathrm{~A} / \mathrm{cm}^{2} \mathrm{~K}^{2}$, which was close to the theoretical value of the $\mathrm{n}$-Si. The interface state densities obtained from the Au/PEDOT:PSS/n-Si SBD decreased with increasing temperature. Furthermore, the conduction mechanism dominating the reverse-bias leakage current in Au/PEDOT:PSS/n-Si SBD was described and discussed. [doi:10.2320/matertrans.M2014263]
\end{abstract}

(Received July 14, 2014; Accepted October 6, 2014; Published November 14, 2014)

Keywords: Schottky barrier diode, poly(3,4-ethylenedioxythiophene):polystyrene sulfonate (PEDOT:PSS), gaussian distribution, barrier inhomogeneity

\section{Introduction}

Schottky barrier diodes (SBDs) have received significant attention in the development of various electronic devices due to their low forward voltage drop and fast switching action. There are numerous reports on the theoretical and experimental studies of SBDs, but they continue to be extensively studied to fully understand the nature of the contact. The fabrication of high quality SBDs with a low ideality factor (n) using a thin interfacial layer is one of the essential prerequisites for electronic devices. Recent research has been performed by applying conducting polymer materials to SBDs as an interfacial layer by a solution based spin-coating process. $^{1-3)}$ It is found that performance, reliability and stability of SBDs depends on various parameters such as the fabrication process, surface preparation, density of surface states distributed at the interface, device temperature and voltage, series resistance $\left(R_{\mathrm{S}}\right)$ of the device, thermal and mechanical stability of the polymer used. Among various conducting polymers, poly(3,4-ethylenedioxythiophene):poly (styrene sulfonate) (PEDOT:PSS), one of the most important polymers, has recently received considerable interest because it exhibits a p-type semiconductor with a high work function $(\sim 5 \mathrm{eV})$, high conductivity $(35-300$ $\mathrm{S} \cdot \mathrm{cm}^{-1}$ at room temperature), good transparency ( $\sim 95 \%$ at visible region), excellent photo-stability, and good environmental stability. ${ }^{4,5)}$ Several research groups have used PEDOT:PSS as a Schottky junction with n-type Si substrates. $^{6-10)}$ According to previous reports, the electrical transport properties of SBDs depend highly on the conditions

*Corresponding author, E-mail: reddy_vrg@rediffmail.com, cjchoi@jbnu. ac.kr of the fabrication processes, such as the utilization of a particular solvent, the amount of PSS, the doping ratio of PEDOT in the PEDOT:PSS mixture, the uniformity of the PEDOT:PSS thin film, and the environmental conditions during the fabrication. As a result, there is a large variation in the electrical transport of PEDOT:PSS/n-Si SBDs. Moreover, there is no experimental study done on their temperature-dependent electrical characteristics. Analysis of the current-voltage $(I-V)$ and capacitance-voltage $(C-V)$ characteristics of metal-semiconductor and metal-insulator-semiconductor structures only at room temperature cannot provide detailed information regarding the current conduction mechanisms or the nature of the barrier formation at the metal-semiconductor interface. ${ }^{11)}$ On the other hand, the temperature dependence of the $I-V$ and $C-V$ characteristics could allow us to gain insight into different aspects of characteristics such as nature of interfacial insulator layer and carrier conduction mechanism.

In this study, we fabricated $\mathrm{Au} /$ n-type $\mathrm{Si} \mathrm{SBDs}$ with a PEDOT:PSS interlayer using spin-coating process and reactive ion etching process (RIE). For the electrical characteristics of the Au/PEDOT:PSS/n-Si SBDs, electrical transport measurement were investigated by both $I-V$ and $C-V$ measurements in the temperature range from 200 to $400 \mathrm{~K}$. The Schottky barrier height $\left(\Phi_{\mathrm{B}}\right), n$, saturation current $\left(I_{0}\right)$, doping concentration $\left(N_{\mathrm{D}}\right)$, and $R_{\mathrm{S}}$ of Au/PEDOT:PSS/n-Si SBDs were extracted at different temperatures. The temperature dependence of the energy distribution of the interface state density $\left(N_{\mathrm{SS}}\right)$ profile was determined based on the variation of the ideality factor with forward bias and temperature. Temperature-dependent barrier characteristics of the Au/PEDOT:PSS/n-Si SBDs were also interpreted based on the existence of the Gaussian distribution of the 
barrier heights. Furthermore, the possible current conduction mechanism of the Schottky contacts modified using the PEDOT:PSS interlayer was also studied.

\section{Experimental Procedures}

The n-type Si (100) wafers with a resistivity of $1-10 \Omega \cdot \mathrm{cm}$ were used as a starting material. The carrier density of Si substrate, measured by Hall measurement system, was extracted to be $1.21 \times 10^{15} \mathrm{~cm}^{3}$. After removing native oxide, the PEDOT:PSS layer was spin-coated on a cleaned $\mathrm{n}$-Si wafer, followed by baking at $150^{\circ} \mathrm{C}$ in air. The scanning electron microscope examination (not shown here) revealed that the thickness of the PEDOT:PSS film was approximately $60 \mathrm{~nm}$. In addition, the sheet resistance of PEDOT:PSS film spin-coated on insulating glass substrate, measured by the four-point probe, was found to be $\sim 2.6 \times 10^{3} \Omega$ /squre. The top $\mathrm{Au}(100 \mathrm{~nm})$ electrode with $1-\mathrm{mm}$ diameter (area $=$ $7.85 \times 10^{-3} \mathrm{~cm}^{2}$ ) was formed on PEDOT:PSS film with a metal mask by an e-beam evaporation system at a pressure of $3.99 \times 10^{-4} \mathrm{~Pa}$. The redundant PEDOT:PSS film was then removed by the $\mathrm{O}_{2}$ plasma RIE process and resulting in a circular dot shaped Au/PEDOT:PSS electrode formed on n$\mathrm{Si}$. The GaIn paste was then applied to the backside of the n-Si wafer for ohmic contacts. The schematic cross section of the $\mathrm{Au} / \mathrm{PEDOT}$ PSS/n-Si SBD is shown in the inset of Fig. 1. The $I-V$ and $C-V$ measurements of the $\mathrm{Au} /$ PEDOT:PSS/n-Si SBD were performed using a semiconductor parameter analyzer (Agilent Technologies, 4155C) and LCR meter (Agilent Technologies, 4284A) at a temperature range of $200-400 \mathrm{~K}$ in steps of $40 \mathrm{~K}$ under a dark condition.

\section{Results and Discussion}

Figure 1 shows the forward and reverse bias semilogarithmic $I-V$ characteristics of the Au/PEDOT:PSS/n-Si SBDs in the temperature range of 200 to $400 \mathrm{~K}$ in $40 \mathrm{~K}$ steps. Sixteen Au/PEDOT:PSS/n-Si SBDs were fabricated and characterized. All diodes exhibited similar electrical characteristics. Furthermore, the $I-V$ characteristics of the fabricated devices was almost identical to that of Au/PEDOT:PSS/n-Si SBDs with the electrode size of 0.5-mm diameter (not shown here), implying that an electrode size dependence of electrical properties of $\mathrm{Au} / \mathrm{PEDOT}: \mathrm{PSS} / \mathrm{n}-\mathrm{Si}$ SBDs was insignificant. In other word, it is obvious that PEDOT:PSS interlayer employed here was uniformly formed in-between $\mathrm{Au}$ film and $\mathrm{Si}$ substrate. The leakage currents of the $\mathrm{Au} /$ PEDOT:PSS/n-Si SBD increases with an increase in temperature and was in the range from $3.89 \times 10^{-12} \mathrm{~A}$ (at $200 \mathrm{~K}$ ) to $2.78 \times 10^{-6} \mathrm{~A}($ at $400 \mathrm{~K}$ ) at $-1 \mathrm{~V}$. As shown in Fig. 1, the diode exhibits rectifying behavior with nonlinear behavior at low voltage, and the current of the diode increases exponentially with increasing voltage, suggesting the diode behaves like a SBD. According to thermionic emission (TE) theory, the current through a SBD at a forward bias $(V \geq 3 k T / q)$ is given by: $\left.{ }^{12}\right)$

$$
I=I_{0} \exp \left(\frac{q V}{n k T}\right)\left[1-\exp \left(\frac{-q V}{k T}\right)\right],
$$

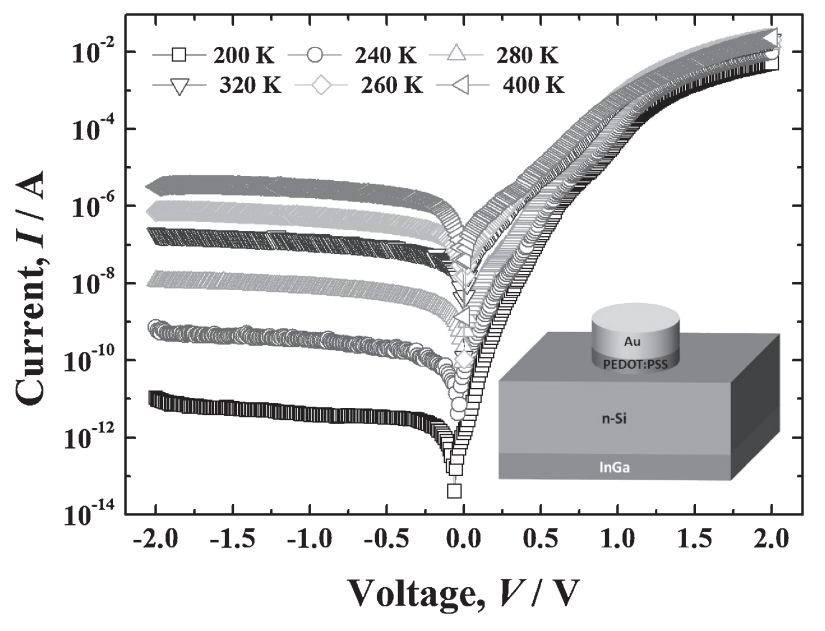

Fig. 1 The forward and reverse bias semi-logarithmic $I-V$ characteristics of the Au/PEDOT:PSS/n-Si SBD in the temperature range of $200-400 \mathrm{~K}$ with $40 \mathrm{~K}$ steps. Inset shows the schematic cross section of the $\mathrm{Au} /$ PEDOT:PSS/n-Si SBD.

where $V$ is the applied bias voltage, $q$ is the electronic charge, $k$ is the Boltzmann constant, $T$ is the absolute temperature in Kelvin, $n$ is the ideality factor, and $I_{0}$ is the saturation current derived from the straight-line intercept of $\ln (I)-V$ at zero bias defined by:

$$
I_{0}=A A^{* *} T^{2} \exp \left(-\frac{q \Phi_{\mathrm{B}}}{k T}\right),
$$

where $A$ is the contact area, $A^{* *}$ is the effective Richardson constant of $120 \mathrm{~A} \mathrm{~cm}^{-2} \mathrm{~K}^{-2}$ for n-type $\mathrm{Si}^{13)}$ and $\Phi_{\mathrm{B}}$ is the zero bias barrier height. Once $I_{0}$ is determined, the Schottky barrier height $\Phi_{\mathrm{B}}(I-V)$ can be evaluated using the following equation:

$$
\Phi_{\mathrm{B}}(I-V)=\frac{k T}{q} \ln \left(\frac{A A^{* *} T^{2}}{k T}\right),
$$

$n$ is introduced for consideration of the deviation of the experimental $I-V$ data from the ideal TE theory and it was obtained from the slope of the linear region of the forward bias $\ln (I)-V$ plot and can be written from eq. (1) as:

$$
n=\frac{q}{k T}\left[\frac{\mathrm{d} V}{\mathrm{~d}(\ln I)}\right]
$$

The experimental values of $\Phi_{\mathrm{B}}$ and $n$ were determined from the intercepts and slopes of the forward bias $\ln I$ versus $V$ plot at each temperature using TE theory. The experimental values of $\Phi_{\mathrm{B}}(I-V)$ and $n$ for the Au/PEDOT:PSS/n-Si SBD were $0.63 \mathrm{eV}$ and 2.75 at $200 \mathrm{~K}$ to $0.97 \mathrm{eV}$ and 1.49 at $400 \mathrm{~K}$, respectively. Figure 2 shows the temperature-dependence of the zero-bias barrier height $(\mathrm{BH})$ and ideality factor $n$. As shown in Fig. 2, the $n$ decreases and the zero bias $\mathrm{BH}$ increases with increasing temperature. Such $n$ behavior can be attributed to the existence of PEDOT:PSS interlayer, particular distribution of $N_{\mathrm{ss}}$ and interface states between a metal and semiconductor. Since the current transport across the contact interface is a temperature activated process, electrons at low temperature are able to overcome the lower barriers and therefore, the current transport will be dominated by the current flowing through the patches of low BHs, 


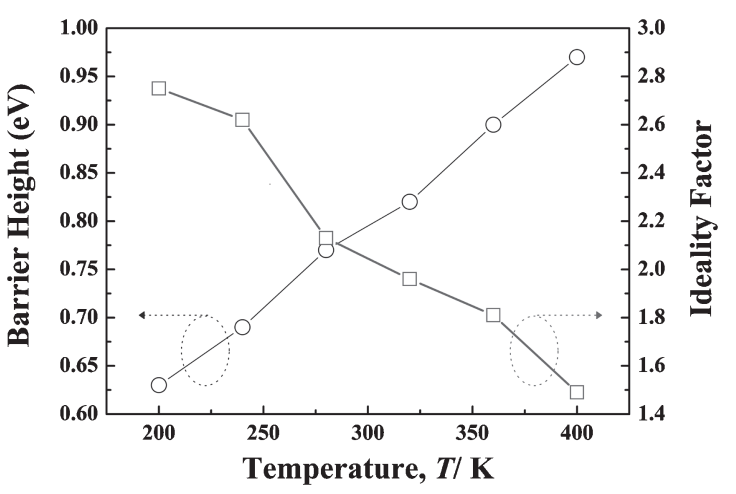

Fig. 2 Temperature dependence of $\Phi_{\mathrm{B}}$ and $n$ obtained from forward bias $I-V$ characteristics for Au/PEDOT:PSS/n-Si in the range of $200-400 \mathrm{~K}$.

leading to a larger $n$. However, as the temperature increases, more electrons have sufficient energy to overcome the $\mathrm{BH}$. An apparent increase in the $n$ and a decrease in the $\mathrm{BH}$ at low temperatures are possibly caused by other effects such as thickness inhomogeneities and non-uniformity of the interfacial charges.

Furthermore, the Au/PEDOT:PSS/n-Si SBD shows a nonideal $I-V$ behavior with a large ideality factor, which may be due to the presence of the PEDOT:PSS interlayer. There are various parameters that cause deviations in ideal behavior including $R_{\mathrm{S}}$ and the presence of an interfacial layer. ${ }^{14)}$ Therefore, $R_{\mathrm{S}}$ of the Au/PEDOT:PSS/n-Si SBD is extracted using a method developed by Cheung and Cheun ${ }^{14}$ at a higher current range. The calculated $R_{\mathrm{S}}$ from the plot of $\mathrm{d} V / \mathrm{d}(\ln I)$ versus $I$ (not shown) were $114.1 \Omega$ at $200 \mathrm{~K}$ and $24 \Omega$ at $400 \mathrm{~K}$ for the Au/PEDOT:PSS $/ \mathrm{n}-\mathrm{Si}$ SBD. The $R_{\mathrm{S}}$ estimated from the plot of $\mathrm{H}(\mathrm{I})$ versus I was $145 \Omega$ at $200 \mathrm{~K}$ and $32.1 \Omega$ at $400 \mathrm{~K}$. The $R_{\mathrm{s}}$ calculated from the $\mathrm{d} V / \mathrm{d}(\ln I)$ versus $I$ plot are in good agreement with those estimated from the $H(I)$ versus $I$ plot, implying their consistency and validity. Experimental results reveal that $R_{\mathrm{S}}$ increases with decreasing temperature. The increase of $R_{\mathrm{S}}$ with a decrease in temperature was due to factors responsible for an increase in $n$ and the lack of free carriers at low temperatures.

Conventional TE theory is typically used to determine the $\mathrm{BH}$. Alternatively, there have been several reports deviating from this classical TE theory. The Richardson plot is drawn to obtain the $\mathrm{BH}$ in another way. Equation (2) can be rewritten as:

$$
\ln \left(\frac{I_{0}}{T^{2}}\right)=\ln \left(A A^{* *}\right)-\frac{q \Phi_{\mathrm{bo}}}{k T},
$$

The conventional Richardson plot of $\ln \left(I_{\mathrm{o}} / T^{2}\right)$ versus $10^{3} / T$ is shown in Fig. 3. The temperature dependence of the experimental $\ln \left(I_{\mathrm{o}} / T^{2}\right)$ versus $10^{3} / \mathrm{T}$ plot produces a straight line. The activation energy and Richardson constant were obtained from the slope and intercept of this straight line as $0.27 \mathrm{eV}$ and $1.98 \times 10^{-7} \mathrm{~A} / \mathrm{cm}^{2} \mathrm{~K}^{2}$, respectively. The experimentally-obtained Richardson constant value was much less than the theoretical Richardson constant value for n-Si. Such a large discrepancy between the experimental and theoretical values of the Richardson constant could be attributed to the calculation using the temperature-dependent $I-V$ characteristics, which may be affected by the lateral inhomogeneity of

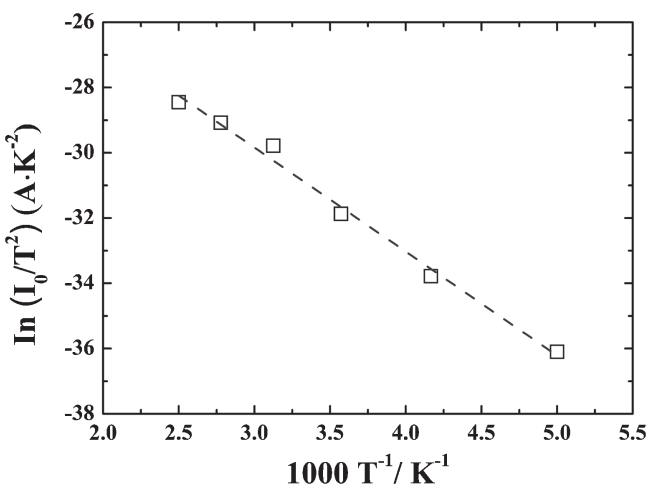

Fig. 3 Richardson plots of the In $\left(I_{0} / T^{2}\right)$ versus $1000 / \mathrm{T}$ for the $\mathrm{Au} /$ PEDOT:PSS/n-Si SBD in the range of $200-400 \mathrm{~K}$.

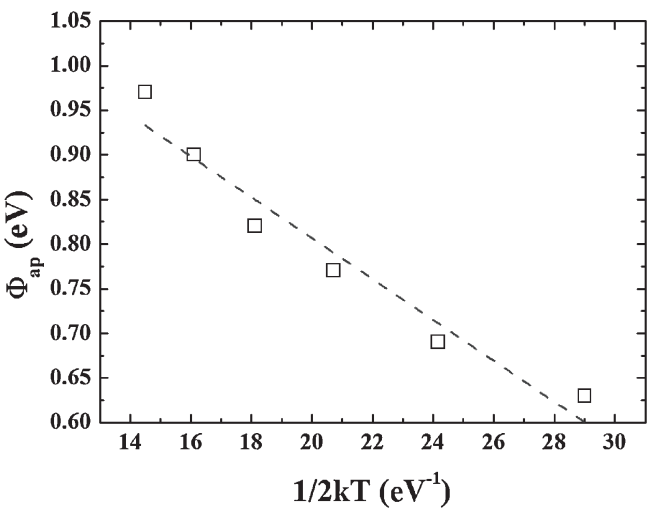

Fig. 4 The zero bias barrier height $\left(\Phi_{\text {ap }}\right)$ versus $1 / 2 k T$ curves of the $\mathrm{Au} /$ PEDOT:PSS SBD according to Gaussian distribution of the barrier height.

the $\mathrm{BH}$ and potential fluctuations due to the PEDOT:PSS interlayer.

In order to explain the abnormal deviations of the $I-V$ characteristics of the SBD from classical TE theory, the lateral distribution of $\mathrm{BH}$ was adopted with a Gaussian distribution. The Gaussian distribution of the $\mathrm{BH}$ with a mean value Schottky barrier height $\left(\bar{\Phi}_{\text {bo }}\right)$ and a standard deviation $\left(\sigma_{\mathrm{o}}\right)$ yields the following expression for the $\left.\mathrm{BH}:{ }^{15}\right)$

$$
\Phi_{\mathrm{ap}}=\overline{\Phi_{\mathrm{bo}}}(T=0)-\frac{q \sigma_{0}^{2}}{2 k T},
$$

where $\Phi_{\text {ap }}$ is the apparent Schottky barrier height and $\sigma_{0}$ is the standard deviation of the $\Phi_{\mathrm{B}}$ distribution that is typically sufficiently small to be neglected. The observed variation of $n$ with temperature in the model is given by: ${ }^{15)}$

$$
\left(\frac{1}{n_{\mathrm{ap}}}-1\right)=\rho_{2}-\frac{q \rho_{3}}{2 k T},
$$

where $n_{\text {ap }}$ is the apparent ideality factor, $\rho_{2}$ and $\rho_{3}$ quantify the voltage deformation of the barrier height distribution, which may depend on temperature. Gaussian distribution may cause the decrease of zero bias $\mathrm{BH}$ and the influence is determined by the standard deviation. As shown in Fig. 4, $\bar{\Phi}_{\mathrm{bo}}$ and $\sigma_{0}$, which correspond to the y-axis intercept and the slope of the linear fit to the plots of $\Phi_{\text {ap }}$ as a function of $1 / 2 \mathrm{kT}$, were estimated as $1.26 \mathrm{eV}$ and $0.15 \mathrm{eV}$, respectively. $\sigma_{0}$ is a general measure of the homogeneity of the Schottky barrier, with a lower $\sigma_{0}$ value corresponding to a more 


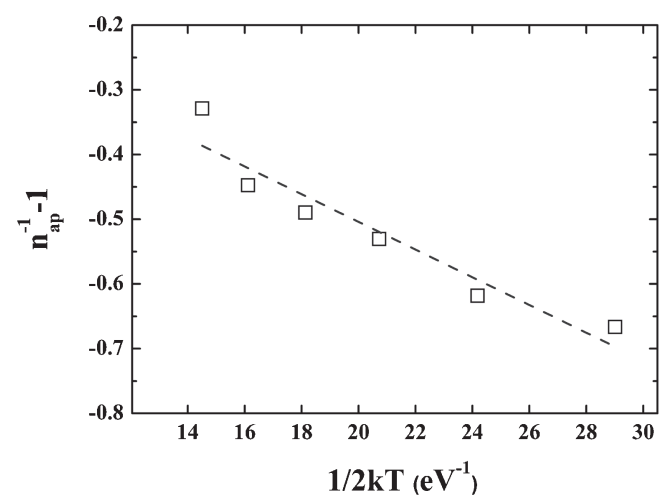

Fig. 5 The ideality factor $\left(n_{\mathrm{ap}}\right)$ versus $1 / 2 k T$ curves of the Au/PEDOT:PSS SBD according to the Gaussian distribution of barrier height.

homogeneous BH. Nevertheless, this inhomogeneity and potential fluctuation dramatically affects the low temperature $I-V$ characteristics. As compared to $\bar{\Phi}_{\mathrm{bo}}(T=0)=1.26 \mathrm{eV}$, the $\sigma_{0}=0.15 \mathrm{eV}$ is not small, implying Schottky barrier inhomogeneity in the studied SBD. As previously shown, ${ }^{15}$ ) barrier inhomogeneities can occur as a result of inhomogeneities in the composition of the interfacial layer, nonuniformity of interfacial charges and interfacial layer thickness. Therefore, low temperature $I-V$ characteristics are affected by this inhomogeneity and potential fluctuation.

Figure 5 shows the plot of $\left(n^{-1}-1\right)$ versus $1 / 2 k T$ for the $\mathrm{Au} / \mathrm{PEDOT}: \mathrm{PSS} / \mathrm{n}-\mathrm{Si}$ SBD. According to eq. (7), this plot should be a straight line that produces the voltage coefficients $\rho_{2}$ and $\rho_{3}$ from the intercept and slope, respectively. Values $\rho_{2}=-0.0757 \mathrm{~V}$ and $\rho_{3}=-0.0214 \mathrm{~V}$ were obtained from the experimental data. The linear behavior of the plot shows that the ideality factor $n$ expresses the voltage deformation of the Gaussian distribution of the SBD. If the value of $\rho_{2}$ is negative, the $\mathrm{BH}$ should decrease with increasing forward bias, which is opposite to image force lowering. The value of $\rho_{3}$ is indeed negative and thus, the $\mathrm{BH}$ increases with increasing forward bias. This bias dependence of $\mathrm{BH}$ in the distribution through mean $\mathrm{BH}$ and standard deviations leads to the temperature dependent ideality factor $n$ in inhomogeneous SBDs. ${ }^{16)}$

The conventional Richardson plot based on the thermionic emission current mechanism exhibits nonlinearity at low temperatures. Considering eqs. (2) and (6), an expression for the modified Richardson plot according to the Gaussian distribution of the BHs can be written as

$$
\ln \left(\frac{I_{0}}{T^{2}}\right)-\left(\frac{q^{2} \sigma_{0}^{2}}{2 k^{2} T^{2}}\right)=\ln \left(A A^{* *}\right)-\frac{q \bar{\Phi}_{\mathrm{bo}}(T=0)}{k T},
$$

According to eq. (8), the modified Richardson plot of $\ln \left(I_{0} /\right.$ $\left.T^{2}\right)-(q \sigma)^{2} / 2(k T)^{2}$ versus $1000 / T$ should be a straight line from which the slope yields the mean $\bar{\Phi}_{\text {bo }}$ and the intercept $\left(\ln A A^{* *}\right)$ at the ordinate determining $A^{* *}$ for a given diode area $A$. As shown in Fig. 6, the modified Richardson plot has quite good linearity over the whole temperature range corresponding to a single activation energy around $\bar{\Phi}_{\mathrm{bo}}$. From this plot, the mean $\bar{\Phi}_{\text {bo }}$ is $1.27 \mathrm{eV}$, which is in good agreement with the mean $\bar{\Phi}_{\text {bo }}(1.26 \mathrm{eV})$ extracted from the plot of $\Phi_{\text {ap }}$ versus $1 / 2 k T$ as shown Fig. 4 . The intercept at the ordinate of the modified $\ln \left(I_{0} / T^{2}\right)-(q \sigma)^{2} / 2(k T)^{2}$ versus

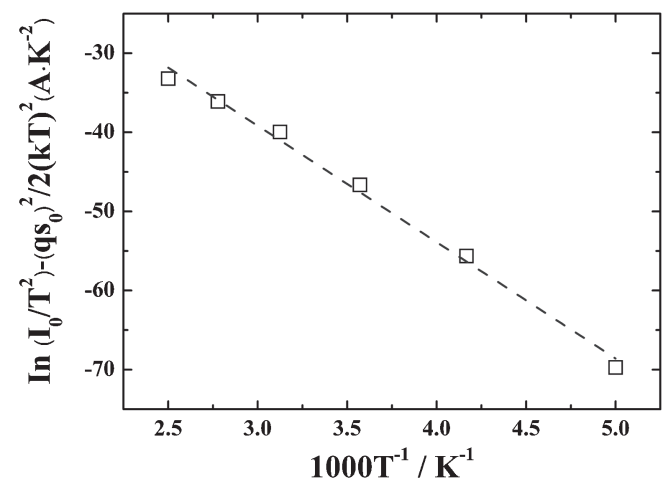

Fig. 6 Modified Richardson $\ln \left(I_{0} / T^{2}\right)-\left(q \sigma_{0}\right)^{2} / 2(k T)^{2}\left(A / K^{2}\right)$ versus $1000 /$ $T$ for the Au/PEDOT:PSS/n-Si SBD.

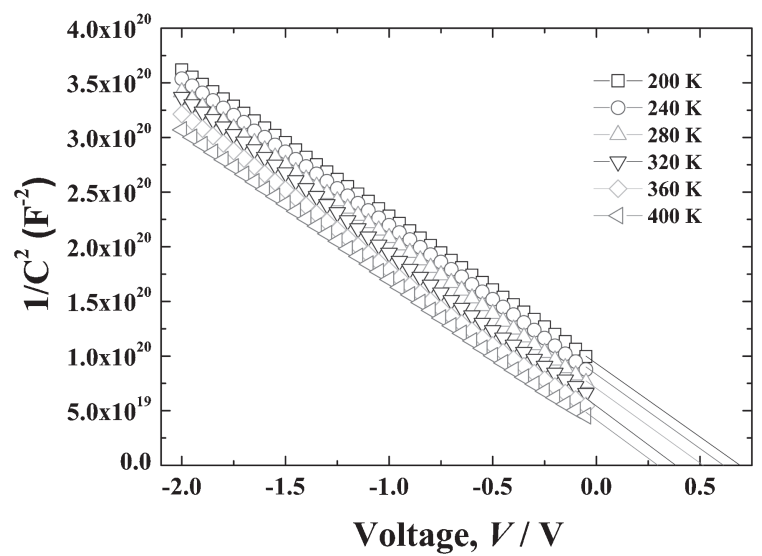

Fig. 7 The reverse bias $C^{-2}-V$ characteristics of the Au/PEDOT:PSS/n-Si SBD at different temperatures in the range $200-400 \mathrm{~K}$ with $40 \mathrm{~K}$ steps.

$1000 / \mathrm{T}$ plot produces a Richardson constant $A^{* *}$ as $134 \mathrm{~A} \mathrm{~K}^{-2} \mathrm{~cm}^{-2}$ without using the temperature coefficient of the BHs. The obtained Richardson constant value is close to the theoretical value of $120 \mathrm{~A} \mathrm{~K}^{-2} \mathrm{~cm}^{-2}$ for $\mathrm{n}-\mathrm{Si}$.

The experimental reverse-bias $C-V$ characteristics of the Au/PEDOT:PSS/n-Si SBD over the temperature range 200$400 \mathrm{~K}$ are shown in Fig. 7. $C-V$ measurements were performed at a frequency of $1 \mathrm{MHz}$. In Schottky diodes, the depletion layer capacitance is expressed as: ${ }^{17)}$

$$
C^{-2}=\left(\frac{2}{\varepsilon_{\mathrm{s}} N_{\mathrm{d}} A^{2}}\right)\left(V_{\mathrm{bi}}-\frac{k T}{q}-V\right),
$$

where $V_{\mathrm{bi}}$ is the built-in voltage, $N_{\mathrm{d}}$ is the donor concentration, $A$ is the area of the Schottky contact, and $\varepsilon_{\mathrm{S}}$ is the dielectric constant of semiconductor $\varepsilon_{\mathrm{s}}=11.9 \varepsilon_{0}$ for $\mathrm{n}-\mathrm{Si},{ }^{18)}$ and $\varepsilon_{0}$ is the permittivity in a vacuum. The built-in voltage $\left(V_{\mathrm{bi}}\right)$ is determined by extrapolating the fitted line of the plotted $C-V$, while $N_{\mathrm{D}}$ was calculated from the slope of the fitted line using the following equation: ${ }^{19)}$

$$
N_{\mathrm{D}}=\frac{2}{q \varepsilon_{\mathrm{s}}}\left[\frac{1}{\mathrm{~d}\left(1 / C^{2}\right) / \mathrm{d} V}\right],
$$

The barrier height $\Phi_{\mathrm{B}}(C-V)$ can be determined by the following equation.:

$$
\Phi_{\mathrm{B}}(C-V)=V_{\mathrm{bi}}+\frac{k T}{q} \ln \left(\frac{N_{\mathrm{C}}}{N_{\mathrm{D}}}\right),
$$


Table 1 Temperature-dependent values of various parameters determined from $I-V$ and $C-V$ characteristics of the Au/PEDOT:PSS/n-Si Schottky barrier diode.

\begin{tabular}{|c|c|c|c|c|c|c|c|}
\hline \multirow[b]{2}{*}{$\begin{array}{c}\text { Temperature, } \\
T / \mathrm{K}\end{array}$} & \multirow[b]{2}{*}{$\begin{array}{c}\text { Current, } \\
I_{\mathrm{o}} / \mathrm{A}\end{array}$} & \multirow[b]{2}{*}{$n$} & \multirow{2}{*}{$\begin{array}{c}\Phi_{\mathrm{B}} \\
(I-V) \\
/ \mathrm{eV}\end{array}$} & \multirow{2}{*}{$\begin{array}{c}\Phi_{\mathrm{B}} \\
(C-V) \\
/ \mathrm{eV}\end{array}$} & \multirow[b]{2}{*}{$N_{\mathrm{D}} / \mathrm{cm}^{-3}$} & \multicolumn{2}{|c|}{$R_{\mathrm{S}} / \Omega$} \\
\hline & & & & & & $\begin{array}{l}\mathrm{d} V / \mathrm{d} \\
(\ln I)\end{array}$ & $H(I)$ \\
\hline 200 & $8.38 \times 10^{-12}$ & 2.75 & 0.63 & 0.87 & $1.32 \times 10^{15}$ & 114.1 & 145.3 \\
\hline 240 & $1.23 \times 10^{-10}$ & 2.62 & 0.69 & 0.83 & $1.34 \times 10^{15}$ & 100.4 & 77.3 \\
\hline 280 & $1.13 \times 10^{-9}$ & 2.13 & 0.77 & 0.78 & $1.35 \times 10^{15}$ & 82.2 & 47.3 \\
\hline 320 & $1.19 \times 10^{-8}$ & 1.96 & 0.82 & 0.69 & $1.36 \times 10^{15}$ & 46.8 & 38.8 \\
\hline 360 & $3.05 \times 10^{-8}$ & 1.81 & 0.90 & 0.64 & $1.37 \times 10^{15}$ & 27.1 & 34.2 \\
\hline 400 & $7.05 \times 10^{-8}$ & 1.49 & 0.97 & 0.63 & $1.41 \times 10^{15}$ & 24.9 & 32.1 \\
\hline
\end{tabular}

$$
\text { with } N_{\mathrm{C}}=2 \frac{\left(2 m^{*} k T\right)^{3 / 2}}{h^{3}}
$$

where $N_{\mathrm{D}}$ is the donor concentration and $N_{\mathrm{C}}$ is the effective density of states in the $\mathrm{Si}$ conduction band edge, $m^{*}=$ $1.08 m_{0}$ is the effective mass of electrons in $\mathrm{Si}$ and $m_{0}$ is the rest mass of the electron. ${ }^{20)}$ The $N_{\mathrm{D}}$ and $\Phi_{\mathrm{B}}(C-V)$ for the $\mathrm{Au} /$ PEDOT:PSS/n-Si SBD can be calculated from eq. (10) and eq. (11), respectively. The values of $\Phi_{\mathrm{B}}(C-V)$ are given in Table 1 . The barrier height values obtained from the reverse bias $C^{-2}-V$ characteristics varied from $0.85 \mathrm{eV}$ at $200 \mathrm{~K}$ to $0.69 \mathrm{eV}$ at $400 \mathrm{~K}$, illustrating $\Phi_{\mathrm{B}}(C-V)$ increases with decreasing temperature. The calculated values of $N_{\mathrm{D}}$ varied from $1.32 \times 10^{15} \mathrm{~cm}^{-3}$ at $200 \mathrm{~K}$ to $1.41 \times 10^{15} \mathrm{~cm}^{-3}$ at $400 \mathrm{~K}$. As shown in Table 1 , the donor concentration of the $\mathrm{n}$-Si slightly increases as the temperature increases. At low temperatures, mostly all of the impurities were frozen out, causing a strong increase in the $R_{\mathrm{S}}$ of the diode, which makes the measured capacitance appear smaller. More electrons may be frozen at the donor level in the freeze-out region and conduction mechanisms in the freeze-out regions are complex. Since the donor concentration increases, then the capacitance also increases with increasing temperature.

Additionally, the barrier heights obtained from $C-V$ measurements were greater than those obtained from $I-V$ measurements. The discrepancy between $\Phi_{\mathrm{B}}(C-V)$ and $\Phi_{\mathrm{B}}(I-V)$ can be attributed to the characteristic nature of the $C-V$ and $I-V$ measurement techniques, and the existence of an interfacial dipole layer or trap states at the PEDOT:PSS $/ n-S i$ interface. Additionally, the discrepancy between $\Phi_{\mathrm{B}}(C-V)$ and $\Phi_{\mathrm{B}}(I-V)$ can be explained by the excess capacitance and SBH inhomogeneities. $^{21)}$ The current in the $I-V$ measurement is dominated by the current that flows through the region of low SBH. Thus, the measured $\Phi_{\mathrm{B}}(I-V)$ is significantly less than the weighed arithmetic average of the SBHs. Alternatively, the barrier height determined from the $C-V$ or flat band was influenced by the distribution of charge at the depletion region boundary, which follows the weighted arithmetic average of the SBHs. Consequently, the SBH calculated from the zero-bias intercept assuming TE as a current transport mechanism is well below the $C-V$ or flat-band measured $\mathrm{BH}$ and the weighted arithmetic average of SBHs. Furthermore, the capacitance $C$ is insensitive to potential fluctuations on a length scale less than the space charge region and the $C-V$ measurement probes the average junction capacitance at the

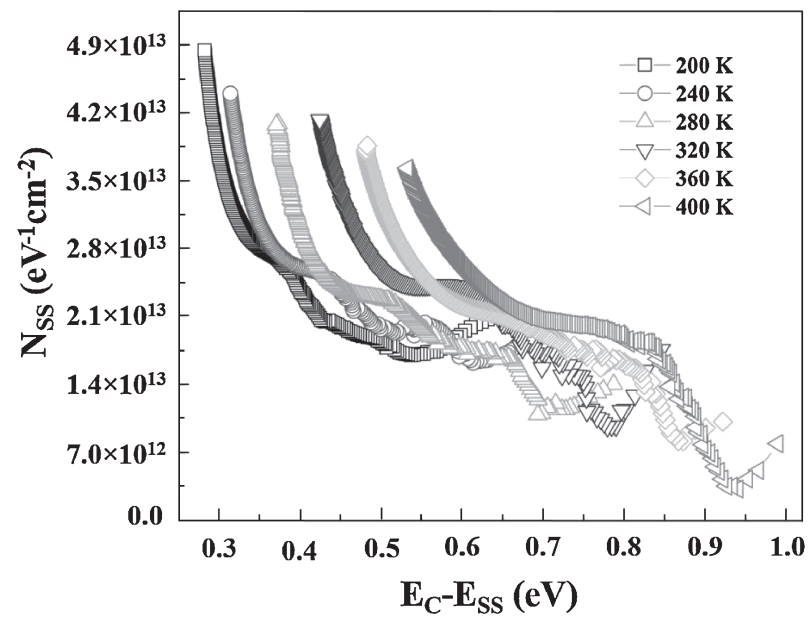

Fig. 8 A plot of $N_{\mathrm{SS}}$ versus $E_{\mathrm{c}}-E_{\mathrm{ss}}$ for the Au/PEDOT:PSS $/ \mathrm{n}-\mathrm{Si}$ SBD with temperatures ranging from 200 to $400 \mathrm{~K}$.

interface, thereby yielding an average value for the barrier height distribution, while the $I-V$ measurement produces a minimum value for the barrier height. ${ }^{22)}$

The $N_{\mathrm{SS}}$ for the Au/PEDOT:PSS/n-Si SBD can be obtained from the forward bias $I-V$ data by taking the voltage-dependent ideality factor $n(V, T)$ with the effective barrier height $\Phi_{\mathrm{b}}(V, T)$. The parameters of $n(V, T)$ and $\Phi_{\mathrm{b}}$ $(V, T)$ can be estimated from the following equations: ${ }^{23)}$

$$
\begin{aligned}
& n(V)=\frac{q}{k T}\left[\frac{V}{\ln \left(I / I_{\mathrm{o}}\right)}\right]=1+\frac{\delta}{\varepsilon_{\mathrm{i}}}\left[\frac{\varepsilon_{\mathrm{S}}}{W_{\mathrm{D}}}+q N_{\mathrm{SS}}\right] \\
& \Phi_{\mathrm{b}}(V, T)=\Phi_{\mathrm{bo}}^{\mathrm{IV}}+\beta V=\Phi_{\mathrm{bo}}^{\mathrm{IV}}+\left(1-\frac{1}{n(V, T)}\right)
\end{aligned}
$$

where $W_{\mathrm{D}}$ is the space charge region width, $\delta$ is the thickness of the interfacial organic layer, $\varepsilon_{\mathrm{i}}$ and $\varepsilon_{\mathrm{S}}$ are the permitivities of the interfacial layer and semiconductor, and $\beta\left(=\mathrm{d} \Phi_{\text {eff }} /\right.$ $\mathrm{d} V=1-1 / n(V))$ is the change in effective $\mathrm{BH}$ with bias voltage. As proposed by Card and Rhoderick, ${ }^{23)}$ in interface states in equilibrium with the semiconductor, the ideality factor becomes greater than unity and the $N_{\mathrm{SS}}$ is given by:

$$
N_{\mathrm{SS}}(V)=\frac{1}{q}\left[\frac{\varepsilon_{\mathrm{i}}}{\delta}(n(V, T)-1)-\frac{\varepsilon_{\mathrm{S}}}{W_{\mathrm{D}}}\right] .
$$

Additionally, in n-type semiconductors, the energy interface states with respect to the conduction band edge $\left(E_{\mathrm{C}}-E_{\mathrm{SS}}\right)$ are obtained as:

$$
E_{\mathrm{C}}-E_{\mathrm{SS}}=q\left(\Phi_{\mathrm{e}}-V\right) .
$$

The voltage dependence of $\mathrm{n}(\mathrm{V})$, and $W_{\mathrm{D}}$ are substituted into eq. (13) to calculate the values of $N_{\mathrm{SS}}$ as a function of $\left(E_{\mathrm{C}^{-}}\right.$ $\left.E_{\mathrm{SS}}\right)$. Figure 8 shows the plot of $N_{\mathrm{SS}}$ as a function of $\left(E_{\mathrm{C}}-E_{\mathrm{SS}}\right)$ for Au/PEDOT:PSS/n-Si SBDs. The $N_{\mathrm{SS}}$ value of $\mathrm{Au} /$ PEDOT:PSS/n-Si SBDs decreases with increasing $E_{\mathrm{C}}-E_{\mathrm{SS}}$ values as a function of temperature. Additionally, the $N_{\mathrm{SS}}$ has exponential growth from the mid gap of $\mathrm{Si}$ toward to the bottom of the conduction band edge for all temperatures. Such behavior of $N_{\mathrm{SS}}$ is a result of molecular restructuring and reordering of PEDOT:PSS and Si molecules at the metal/ semiconductor interface under the influence of temperature. $^{24)}$ The $N_{\mathrm{SS}}$ is in the range of $1.78 \times 10^{13} \mathrm{eV}^{-1} \mathrm{~cm}^{-2}$ at $200 \mathrm{~K}$ to $4.8 \times 10^{12} \mathrm{eV}^{-1} \mathrm{~cm}^{-2}$ at $400 \mathrm{~K}$. These values are 
similar to the results reported in the literature. ${ }^{25,26)}$ The interface states and interfacial layer between the metal and semiconductor play a significant role in the determination of the Schottky barrier parameters of the devices.

The current transport mechanism dominating the reverse leakage current in the Au/PEDOT:PSS/n-Si SBD was then investigated using the electric field dependence by considering Poole-Frenkel emission and Schottky emission across the junction. The $I-V$ expression for the Poole-Frenkel effect are given as: ${ }^{27,28)}$

$$
I_{\mathrm{R}}=I_{\mathrm{o}} \exp \left(\frac{\beta_{\mathrm{PF}}}{k_{\mathrm{B}} T} \sqrt{E}\right),
$$

and for the Schottky effect,

$$
I_{\mathrm{R}}=A A^{*} T^{2} \exp \left(\frac{\beta_{\mathrm{S}}}{k_{\mathrm{B}} T} \sqrt{E}\right)
$$

where $\beta_{\mathrm{PF}}$ and $\beta_{\mathrm{S}}$ are the Poole-Frenkel and Schottky fieldlowering coefficients, respectively. The theoretical values for $\beta_{\mathrm{PF}}$ and $\beta_{\mathrm{S}}$ are given by:

$$
2 \beta_{\mathrm{S}}=\beta_{\mathrm{PF}}=\left(\frac{q^{3}}{\pi \varepsilon}\right)^{1 / 2} .
$$

The Poole-Frenkel and Schottky effects can typically be distinguished by values of the field-lowering coefficients. The theoretical values of the Poole-Frenkel field-lowering coefficient $\left(\beta_{\mathrm{PF}}\right)$ and Schottky field-lowering coefficient $\left(\beta_{\mathrm{S}}\right)$ for the $\mathrm{Au} / \mathrm{PEDOT}: \mathrm{PSS} / \mathrm{n}-\mathrm{Si} \mathrm{SBD}$ were $\beta_{\mathrm{PF}}=2.22 \times 10^{-5}$ $\mathrm{eV} / \mathrm{m}^{1 / 2} \mathrm{~V}^{-1 / 2}$ and $\beta_{\mathrm{S}}=1.11 \times 10^{-5} \mathrm{eV} / \mathrm{m}^{1 / 2} \mathrm{~V}^{-1 / 2}$. The $\beta_{\mathrm{PF}}$ value is always twice the value of $\beta_{\mathrm{S}}$. Figure 9 shows the plot of $\ln \left(I_{\mathrm{R}}\right)$ versus $E^{1 / 2}$ for Au/PEDOT:PSS/n-Si SBDs at different temperatures. The slopes determined from the fit to the data are $2.58 \times 10^{-5}, 2.44 \times 10^{-5}, 2.39 \times 10^{-5}, 2.28 \times$ $10^{-5}, 1.25 \times 10^{-5}$ and $1.24 \times 10^{-5} \mathrm{eV} / \mathrm{m}^{1 / 2} \mathrm{~V}^{-1 / 2}$ for 200 , $240,280,320,360$, and $400 \mathrm{~K}$ respectively. By comparing the experimental and theoretical slopes of Au/PEDOT:PSS/ n-Si SBDs, the experimental slope determined in the low temperature region $(T<320)$ was closer to the Poole-Frenkel field-lowering coefficient $\left(\beta_{\mathrm{PF}}\right)$, whereas the experimental slopes in the higher temperature region $(T>320)$ was closer to the Schottky field-lowering coefficient $\left(\beta_{\mathrm{S}}\right)$. This indicates that Poole-Frenkel emission is dominant in the lower temperature region while Schottky emission is dominant in the higher temperature region. Therefore, the current conduction mechanism of Au/PEDOT:PSS/n-Si SBDs changed from Poole-Frenkel emission to Schottky emission in the temperature range of $320-360 \mathrm{~K}$.

\section{Conclusions}

The Au/PEDOT:PSS/n-Si SBDs were fabricated by simple spin-coating technique and sequential RIE process. The influence of a thin PEDOT:PSS interlayer on the electrical properties of $\mathrm{Au} / \mathrm{n}$-type Si SBDs were studied in the temperature range of $200-400 \mathrm{~K}$ by $I-V$ and $C-V$ measurements. The values of $\Phi_{\mathrm{B}}(I-V), \Phi_{\mathrm{B}}(C-V), n$ and $R_{\mathrm{S}}$ of $\mathrm{Au} / \mathrm{PEDOT}: \mathrm{PSS} / \mathrm{n}-\mathrm{Si}$ SBDs were estimated and illustrated to be strongly temperature dependent. The investigation results revealed an abnormal decrease in the zero-bias $\mathrm{BH}$ and an increase in the ideality factor $n$ with decreasing

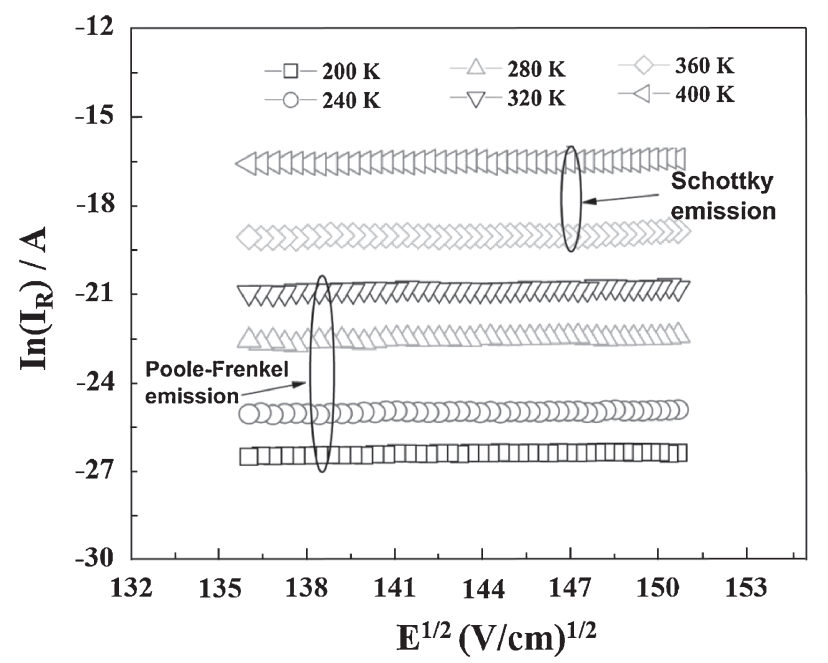

Fig. 9 Reverse current (in logarithmic scale) versus $E^{1 / 2}$ plots for the $\mathrm{Au} /$ PEDOT:PSS/n-Si SBD at different temperatures.

temperature. Additionally, the electrical properties confirmed that the temperature-dependent $I-V$ characteristics of $\mathrm{Au} /$ PEDOT:PSS/n-Si SBDs can be explained on the basis of TE theory with a Gaussian distribution of BHs. The Gaussian distribution has a mean $\mathrm{BH} \bar{\Phi}_{\text {bo }}$ of $1.26 \mathrm{eV}$ and a standard deviation $\left(\sigma_{\mathrm{o}}\right)$ of $0.15 \mathrm{eV}$. The extracted values of $\bar{\Phi}_{\mathrm{bo}}$ and $\sigma_{\mathrm{o}}$ clearly indicates the presence of Schottky barrier inhomogeneities in the present device. A modified $\ln \left(I_{0} / T^{2}\right)-$ $\left(q^{2} \sigma_{0}^{2} / 2 k^{2} T^{2}\right)$ versus $1000 / \mathrm{T}$ plot produces $\bar{\Phi}_{\mathrm{bo}}$ and $A^{* *}$ as $1.27 \mathrm{eV}$ and $134 \mathrm{~A} / \mathrm{cm}^{2} \mathrm{~K}^{2}$. The obtained $A^{* *}$ value is in close agreement with the known value of $120 \mathrm{~A} / \mathrm{cm}^{2} \mathrm{~K}^{2}$ for $\mathrm{n}-\mathrm{Si}$. The energy distribution profile of $N_{\mathrm{SS}}$ is obtained from the forward bias $I-V$ characteristics by considering the bias dependence of the effective $\mathrm{BH}\left(\Phi_{\mathrm{e}}\right)$ and ideality factor $(n(\mathrm{~V}))$, and the $N_{\mathrm{SS}}$ values decreases with an increase in temperature (from $1.78 \times 10^{13} \mathrm{eV}^{-1} \mathrm{~cm}^{-2}$ at $200 \mathrm{~K}$ to $4.8 \times$ $10^{12} \mathrm{eV}^{-1} \mathrm{~cm}^{-2}$ at $400 \mathrm{~K}$ ). Additionally, the experimental results showed that the Poole-Frenkel emission is the dominant mechanism in the reverse leakage current at low temperatures (200-320 K), whereas Schottky emission is the dominant mechanism at high temperatures (360-400 K).

\section{Acknowledgements}

This research was supported by Basic Research Laboratory Program (2011-0027956) through the National Research Foundation of Korea funded by the Ministry of Education, Science and Technology, and by the Converging Research Center Program (2014M3C1A8048834) through the Ministry of Science, ICT \& Future Planning, Republic of Korea. It was also supported by the R\&D Program (Grant No. 10045216) for Industrial Core Technology funded by the Ministry of Trade, Industry and Energy (MOTIE), Republic of Korea.

\section{REFERENCES}

1) S. Demirezen, Z. Sönmez, U. Aydemir and S. Alrindal: Curr. Appl. Phys. 12 (2012) 266-272.

2) K. Akkılıç, M. E. Aydın, I. Uzun and T. Kılıçoglu: Synth. Met. 156 (2006) 958-962. 
3) N. Șimșir, H. Șafak, Ö. F. Yüksel and M. Kuș: Curr. Appl. Phys. 12 (2012) $1510-1514$

4) K. S. Kang, K. J. Han and J. Kim: IEEE Trans. Nanotechn. 8 (2009) 627-630.

5) W. Feng, Y. Li, J. Wu, H. Noda, A. Fujii, M. Ozaki and K. Yoshino: J. Phys. Condens. Mater. 19 (2007) 186220.

6) Z. Tang, Q. Liu, I. Khatri, R. Ishikawa, K. Ueno and H. Shirai: Phys. Status Solidi C 9 (2012) 2075-2078.

7) Y. Lin and Y. Su: J. Appl. Phys. 111 (2012) 073712.

8) Y. Lin, B. Huang, Y. Lien, C. Lee, C. Tsai and H. Chang: J. Phys. D: Appl. Phys. 42 (2009) 165104.

9) M. Price, J. Foley, R. May and S. Maldonado: Appl. Phys. Lett. 97 (2010) 083503

10) J. Lin, J. Zeng, Y. Su and Y. Lin: Appl. Phys. Lett. 100 (2012) 153509.

11) T. Tunç, I. Dökme, Ș. Altindal and I. Uslu: Optoelectron. Adv. Mater. Rapid Commun. 4 (2010) 947-950.

12) V. Janardhanam, H.-K. Lee, K.-H. Shim, H.-B. Hong, S.-H. Lee, K.-S. Ahn and C.-J. Choi: J. Alloy. Compd. 504 (2010) 146-150.

13) E. H. Rhoderick and R. H. Williams: Metal-Semiconductor Contacts 2nd ed., (Clarendon Press, Oxford; U.K., 1988) pp. 38-41.

14) S. K. Cheung and N. W. Cheung: Appl. Phys. Lett. 49 (1986) 85-87.

15) Y. Song, R. Van Meirhaeghe, W. Laflere and F. Cardon: Solid State
Electron. 29 (1986) 633-638.

16) N. Yıldırım and A. Türüt: Microelectron Eng. 86 (2009) 2270-2274.

17) S. Chand and S. Bala: Appl. Surf. Sci. 252 (2005) 358-363.

18) S. Ashok, J. M. Borrego and R. J. Gutmann: Solid-State Electron. 22 (1979) 621-631.

19) R. T. Tung: Phys. Rev. B 45 (1992) 13509-13523.

20) S. Chand and J. Kumar: J. Semicond. Sci. Technol. 11 (1996) 12031208.

21) F. Yakuphanoglu and B. F. Senkel: J. Phys. Chem. C 111 (2007) 18401846.

22) Ö. Güllü, O. Baris, M. Biber and A. Türüt: Appl. Surf. Sci. 254 (2008) 3039-3044.

23) H. C. Card and E. H. Rhoderick: J. Phys. D: Appl. Phys. 4 (1971) 1589-1601.

24) B. Akkal, Z. Benamara, A. Boudissa, N. B. Bouiadjra, M. Amrani, I. Bideux and B. Gruzza: Mater. Sci. Eng. B 55 (1998) 162-168.

25) O. Pakma, N. Serin, T. Serin and S. Altindal: Semicond. Sci. Technol. 23 (2008) 105014.

26) F. Z. Pür and A. Tataroğlu: Phys. Scr. 86 (2012) 035802.

27) W. Z. Schottky: Physik. 15 (1914) 872-878.

28) J. Frenkel: Phys. Rev. 54 (1938) 647-648. 